Internist 2006 47:853-856

DOI 10.1007/s00108-006-1632-9

Online publiziert: 30. Mai 2006

๑) Springer Medizin Verlag 2006

\section{Rubrikherausgeber}

K. Werdan, Halle/Saale
M. Degonda Halter ${ }^{3}$ P. Nebiker ${ }^{1}$ B. Hug ${ }^{1} \cdot$ M. Oberholzer ${ }^{2}$. U. Flückiger ${ }^{3}$.

S. Bassetti ${ }^{3}$

${ }^{1}$ Medizinische Klinik B, Universitätsspital Basel

${ }^{2}$ Institut für Pathologie, Universität Basel

${ }^{3}$ Klinik für Infektiologie und Spitalhygiene, Universitätsspital, Basel

\title{
Atypisches Erythema induratum Bazin bei tuberkulöser Osteomyelitis
}

\section{Fallbericht \\ Vorgeschichte}

Eine 79-jährige Patientin mit einer bekannten schweren sensomotorischen Polyneuropathie unklarer Ätiologie und einer Chondrokalzinose der Knie wurde im November 2004 zur Abklärung eines Status febrilis mit Zunahme von „multiplen subkutanen Knoten“ stationär aufgenommen.

Im Juli 2004 war eine Bursitis am linken Ellbogen und eine Lymphadenopathie axillär links aufgetreten. Zwei dieser axillären Lymphknoten waren exstirpiert worden. Histologisch hatten sich ausgedehnte verkäsende Nekrosen gezeigt, umgeben von einem Epitheloidzellsaum und mehrkernigen Riesenzellen vom Langhans-Typ. Es waren keine Kulturen der Lymphknoten angelegt worden, eine Polymerasekettenreaktion- (PCR-)Untersuchung auf Mycobacterium tuberculosis im exzidierten Material war vom Labor als „nicht beurteilbar“ abgegeben worden. Nach initial spontaner Regredienz war es im September 2004 zu einem Rezidiv der Bursitis mit einer neu aufgetretenen Fistel über dem linken Olekranon gekommen, welche auch nach einer 10-tägigen antibiotischen Therapie persistierte. In der Folge traten 5 rundliche subkutane Raumforderungen am Stamm und am linken Oberarm auf, mit einem Durchmesser von $2-5 \mathrm{~cm}$ (• Abb. 1).
Die Patientin gab einen Gewichtsverlust von $7 \mathrm{~kg}$ in 6 Monaten an, ohne Fieber und ohne begleitenden Husten oder Nachtschweiß. Eine durchgemachte Tuberkulose oder andere Risikofaktoren (Exposition, Herkunft, Immunsuppression) lagen nicht vor. Als Komorbiditäten bestanden eine Polyneuropathie und eine proximale Myopathie der Beine unklarer Ätiologie sowie ein tachykardes Vorhofflimmern.

\section{Klinischer Befund}

Die febrile Patientin (Körpertemperatur $38,6^{\circ} \mathrm{C}$ ) war in leicht reduziertem Allgemeinzustand. Es fanden sich 5 subkutane Raumforderungen mit einem Durchmesser von 2-5 cm (• Abb. 1). Zwei dieser Raumforderungen lagen am linken Oberarm über der V. brachialis, eine kranial der linken Mamma und 2 am Rücken lateral links. Sie waren prallelastisch, nicht gerötet und indolent. Das übrige Integument sowie der Lymphknotenstatus waren bis auf axilläre Narben nach Lymphknotenexzision unauffällig. Kardiopulmonal war die Patientin kompensiert. Wegen der bekannten Polyneuropathie und Myopathie der Beine war die Patientin nur noch am Rollator gehfähig.

\section{Diagnostik}

Labor. Leukozyten $7,3 \mathrm{G} / 1$ (Normalwerte: 3,5-10,o G/l), CRP $28 \mathrm{mg} / \mathrm{l}$ (<10 mg/l), BSG $42 \mathrm{~mm} / \mathrm{h}(<29 \mathrm{~mm} / \mathrm{h})$, Hämoglobin

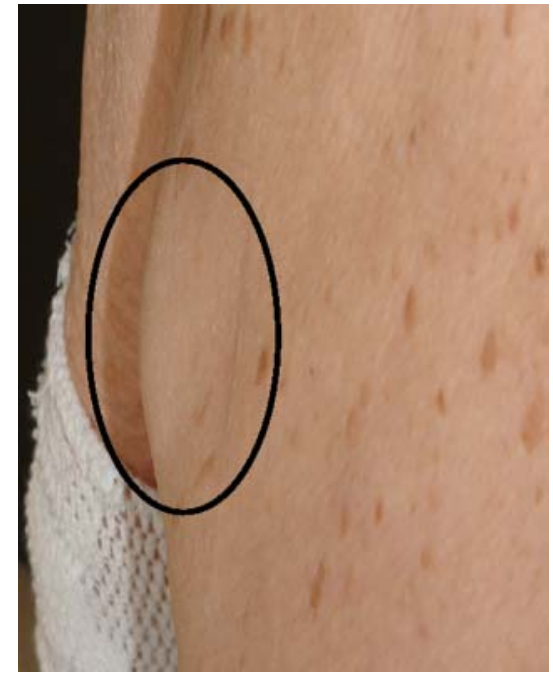

Abb. 1 A Tuberkulid links dorsolateral

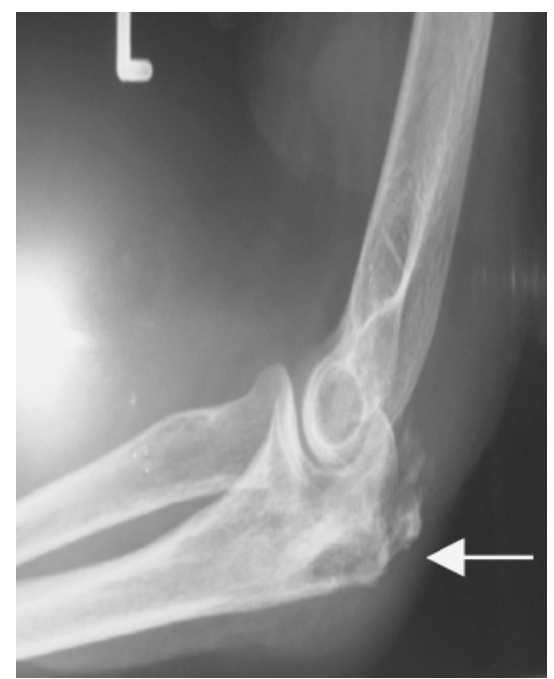

Abb. 2 A Osteolyse am linken Olekranon 


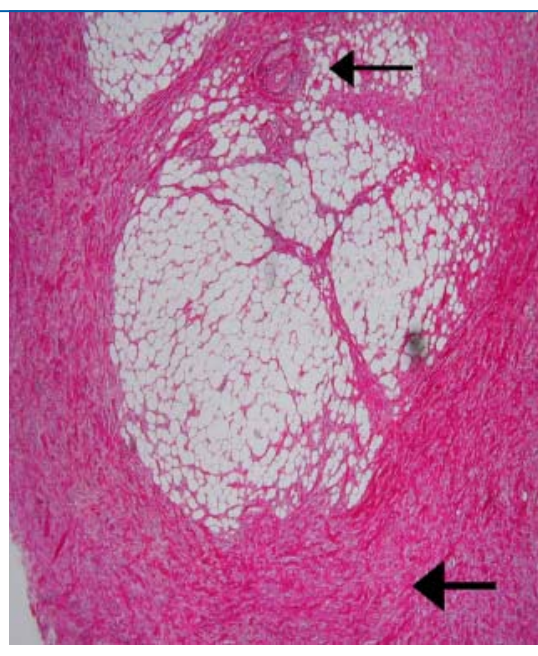

Abb. $3 \Delta$ Histologie des exzidierten Knotens: Epitheloid-riesenzellig nekrotisierende Entzündung mit Pannikulitis und Begleitvenulitis. [H.E.Färbung, 1:2,5. Kleiner Pfeil: kleine Vene mit Entzündung. Großer Pfeil: starke Vermehrung und Sklerosierung des Bindegewebes in der Subkutis. Das Bindegewebe umscheidet das Fettgewebe (im Zentrum), sodass die Fettgewebeläppchen wie kleine Inseln erscheinen.]

$102 \mathrm{~g} / \mathrm{l}(120-160 \mathrm{~g} / \mathrm{l})$, Leber- und Nierenwerte im Normbereich.

Bildgebung. Das Röntgenbild des linken Ellbogens ( $\bullet$ Abb. 2) zeigte eine Osteolyse mit Randsklerosierung im Bereich des Olekranons und unregelmäßig begrenzte röntgendichte Strukturen kranial des Olekranons (DD: Verkalkungen im Bereich des Trizepssehnenansatzes). Im Thoraxröntgenbild war das Lungenparenchym unauffällig.

\section{Mantoux-Test. Negativ.}

Bakteriologie. Kulturen für aerobe und anaerobe Bakterien, Direktpräparate auf säurefeste Stäbchen und PCR-Untersuchungen auf Mycobacterium-tuberculosis-Komplex waren negativ aus $2 \mathrm{Ab}$ strichen der Fistelöffnung sowie aus Abstrichen und Biopsien von 2 subkutanen Knoten, die kranial der linken Mamma und am linken Oberarm exzidiert wurden. Schließlich wurde Flüssigkeit aus der Fistel aspiriert. In diesem Aspirat konnten bereits im Direktpräparat vereinzelt säurefeste Stäbchen nachgewiesen werden, die PCR-Untersuchung auf M.-tuberculosisKomplex war positiv. 4-6 Wochen später wurde M. tuberculosis (sensibel auf alle getestete Tuberkulostatika) auch kulturell im Fistelaspirat, in den Abstrichen und
Biopsien der subkutanen Knoten und in einem der beiden Abstriche der Fistelöffnung nachgewiesen.

Histologie. Die Biopsien der exzidierten subkutanen Knoten zeigten eine epitheloid riesenzellig nekrotisierende Pannikulitis mit Vaskulitis, typisch für ein Erythema induratum Bazin (noduläre Vaskulitis; • Abb. 3).

\section{Diagnose}

\section{- Erythema induratum Bazin (noduläre Vaskulitis) bei tuberkulöser Osteomyelitis des linken Olecranons ulnae.}

\section{Therapie und Verlauf}

Wegen der vorbestehenden schweren Polyneuropathie musste eine tuberkulostatische Therapie ohne Isoniazid gewählt werden. Deswegen wurde resistenzgerecht eine Behandlung mit Rifampicin, Pyrazinamid und Ethambutol über 12 Monate durchgeführt. Unter der Therapie verbesserte sich der Allgemeinzustand der Patientin, die Sekretion aus der Fistel versiegte allmählich. Zwei Monate nach Beginn der Behandlung war keine Fistelöffnung mehr erkennbar. Die subkutanen Knoten waren nach 4 Monaten immer noch vorhanden. Nach Abschluss der einjährigen Therapie waren sie nicht mehr nachweisbar.

\section{Diskussion}

\section{Hauttuberkulose}

Die Hauttuberkulose ist mit einer Prävalenz von $1,5 \%$ aller extrapulmonalen $\mathrm{Tu}$ berkulosefälle in Europa und den USA eine insgesamt seltene klinische Entität [4]. Durch die Zunahme der TuberkuloseInzidenz, welche mit der pandemischen Ausbreitung der HIV-Infektion assoziiert ist, gewinnt sie jedoch an Bedeutung im Bezug auf differenzialdiagnostische Überlegungen bei unklaren Hautmanifestationen [5].

Die Hauttuberkulosen wurden ursprünglich in 2 Gruppen unterteilt:
- Formen, bei denen Mykobakterien in den Hautläsionen nachgewiesen werden („echte“ Hauttuberkulosen),

- Formen ohne Erregernachweis (Tuberkulide), die als parainfektiöse Mitreaktion bei Organtuberkulosen interpretiert wurden.

Die „echten“ Hauttuberkulosen können weiter nach dem Infektionsweg (exogen, endogen) eingeteilt werden ( $\bullet$ Tab. 1 ; [1]). Die Gültigkeit dieser Einteilungen wird aber durch Resultate von Untersuchungen mit neueren und sensitiveren kulturellen und diagnostischen Methoden (z. B. PCR-Untersuchungen) zunehmend in Frage gestellt.

Tuberkulide gehören zu den häufigsten Formen der Hauttuberkulose. In industrialisierten asiatischen Ländern wie Japan und Hongkong nimmt ihre Inzidenz zu, im Gegensatz zur Inzidenz der „echten“ Hauttuberkulosen [3, 6]. Es wird postuliert, dass Tuberkulide Folge einer Hypersensitivitätsreaktion sind, welche durch hämatogene Streuung von Antigenfragmenten von toten Mykobakterien entsteht.

\section{Erythema induratum Bazin}

Das Erythema induratum Bazin (EIB; noduläre Vaskulitis) ist das häufigste Tuberkulid $[3,6]$ und tritt überwiegend bei Frauen in mittlerem und fortgeschrittenem Alter auf. Histologisch handelt es sich um eine lobuläre Pannikulitis mit Vaskulitis. Von den meisten Autoren werden die Begriffe „Erythema induratum Bazin“ und "noduläre Vaskulitis“ synonym gebraucht. Die Assoziation zwischen EIB und Tuberkulose wird kontrovers diskutiert, vor allem nachdem PCR-Untersuchungen gezeigt haben, dass DNA von M. tuberculosis nur in $14-77 \%$ der EIB-Läsionen nachweisbar ist und dass in gewissen Studien bis zu $60 \%$ der Patienten einen negativen Tuberkulintest hatten. Man geht heute davon aus, dass neben der Tuberkulose auch andere Ursachen des EIB existieren müs$\operatorname{sen}[7,8]$.

Das EIB manifestiert sich typischerweise mit bilateralen, erythematösen, meistens druckdolenten, subkutanen Knoten an den dorsalen Unterschenkeln, die nicht selten auch exulzerieren und vernarben 
Tab. 1 Einteilung der Hauttuberkulosen

\begin{tabular}{|c|c|c|}
\hline \multirow{2}{*}{\multicolumn{2}{|c|}{$\begin{array}{l}\text { Krankheit } \\
\text { „Echte“ Hauttuberkulosen }\end{array}$}} & \multirow[t]{2}{*}{ Infektionsweg } \\
\hline & & \\
\hline \multirow{3}{*}{$\begin{array}{l}\text { - Mit exo- } \\
\text { gener Infek- } \\
\text { tion }\end{array}$} & Tuberkulöser Schanker & Direkte Inokulation \\
\hline & Tuberculosis verrucosa cutis & Direkte Inokulation \\
\hline & Lupus vulgaris (einige Fälle) & $\begin{array}{l}\text { Direkte Inokulation } \\
\text { BCG-Impfung }\end{array}$ \\
\hline \multirow{6}{*}{$\begin{array}{l}\text { - Mit en- } \\
\text { dogener } \\
\text { Infektion }\end{array}$} & Lupus vulgaris (die meisten Fälle) & $\begin{array}{l}\text { Hämatogen, lymphatisch, per } \\
\text { continuitatem }\end{array}$ \\
\hline & Skrofuloderm & Per continuitatem \\
\hline & Miliartuberkulose der Haut & Hämatogen \\
\hline & Periorifizielle Tuberkulose & Autoinokulation \\
\hline & Tuberkulöser Abszess & Hämatogen \\
\hline & Papulonekrotisches Tuberkulid (die meisten Fälle) & Hämatogen \\
\hline \multicolumn{3}{|l|}{ Tuberkulide } \\
\hline & Lichen scrofulosorum & Hämatogen \\
\hline & Erythema induratum Bazin (noduläre Vaskulitis) & Hämatogen \\
\hline & Noduläre granulomatöse Phlebitis & Hämatogen \\
\hline & Papulonekrotisches Tuberkulid (einige Fälle) & Hämatogen \\
\hline
\end{tabular}

$[1,2,7]$. Das EIB ist häufiger bei adipösen Frauen mit chronischer venöser Insuffizienz der unteren Extremitäten, verläuft chronisch-rezidivierend, und spricht nur langsam auf die Therapie an. Rezidive und Ulzerationen der Knoten treten oft während den kalten Jahreszeiten auf.

Beim typischen EIB, wie bei allen Tuberkuliden, sind Mykobakterien weder im Direktpräparat noch in den Kulturen der Hautläsionen nachweisbar, der Tuberkulintest ist in der Regel deutlich positiv. Die Diagnose wird histologisch gestellt. Die Therapie besteht in der tuberkulostatischen Behandlung der assoziierten Tuberkulose [7].

\section{Atypische Präsentationen des Erythema induratum Bazin}

Alle Formen der Hauttuberkulose können sich mit sehr unterschiedlichen klinischen Bildern manifestieren. Auch atypische Formen des EIB sind in der Literatur beschrieben. Dabei handelt es sich z. B. um EIB bei Männern, EIB mit negativem Tuberkulintest, oder EIB mit atypischer Lokalisation (z. B. unilateral, Oberschenkel).

Unsere Patientin präsentierte sich ebenfalls mit einer atypischen Klinik (reizlose Knoten an der oberen linksseitigen Körperhälfte) und einem negativen Tuberkulintest. Außerordentlich, und unseres Wissens noch nie beschrieben, ist aber die Tatsache, dass in den Abstrichen und Biopsien der subkutanen EIB-Knoten kulturell M. tuberculosis nachgewiesen werden konnte. Das unterstützt die Hypothese, dass Tuberkulide und insbesondere das EIB unterschiedliche Pathogenesen haben können und dass das EIB nicht nur durch eine Hypersensitivitätsreaktion, sondern auch durch eine hämatogene oder lymphogene Streuung von lebenden $\mathrm{M}$. tuberculosis entstehen kann.

\section{Fazit für die Praxis}

1. Bei unklaren Lymphadenopathien sollten exzidierte Lymphknoten immer auch bakteriologisch untersucht werden (einschließlich der Kultur auf Mykobakterien).

2. Alle Formen der Hauttuberkulose können sich mit sehr unterschiedlichen klinischen Bildern manifestieren. Die Diagnose kann deshalb schwierig sein. Bei unklaren Hautläsionen, insbesondere wenn nodulär und/oder exulzerierend, muss differenzialdiagnostisch an eine Tuberkulose gedacht werden.

3. Das Erythema induratum Bazin könnte verschiedene Pathogenesen haben (Hypersensitivitätsreaktion, hämatogene Streuung).
Internist 2006 - 47:853-856

DOI 10.1007/s00108-006-1632-9

○) Springer Medizin Verlag 2006

M. Degonda Halter - P. Nebiker - B. Hug • M. Oberholzer - U. Flückiger · S. Bassetti Atypisches Erythema induratum Bazin bei tuberkulöser Osteomyelitis

\section{Zusammenfassung}

Hauttuberkulosen können sich mit sehr unterschiedlichen klinischen Bildern manifestieren und damit die Diagnosestellung erschweren. Wir stellen den Fall einer 79-jährigen Patientin vor, mit einer atypischen Präsentation eines Erythema induratum Bazin (EIB) am Oberkörper und einer tuberkulösen Osteomyelitis des Olekranon links. Aus den Biopsien der EIB-Knoten konnte M. tuberculosis kulturell nachgewiesen werden. Das widerspricht der klassischen Vorstellung, dass das EIB als Folge einer Hypersensitivitätsreaktion auf Mykobakterien entsteht, und unterstützt die Hypothese, dass das EIB auch durch eine hämatogene oder lymphogene Streuung von lebenden $M$. tuberculosis entstehen kann.

Schlüsselwörter

Erythema induratum Bazin · Noduläre Vaskulitis · Tuberkulid · Hauttuberkulose · Tuberkulose

\section{Atypical erythema induratum Bazin with tuberculous osteomyelitis}

\section{Abstract}

Diagnosis of all types of cutaneous tuberculosis is challenging because the clinical picture of these diseases is highly variable. We describe the case of a 79-year old woman with an atypical presentation of Erythema induratum Bazin (EIB) on the chest and left arm in association with a tuberculous osteomyelitis of the left olecranon. Surprisingly, M. tuberculosis grew also from biopsies of the EIB-lesions. This contradicts the conventional view that considers EIB (a tuberculid) to be caused by a hypersensitivity reaction to mycobacteria. The presented case supports the hypothesis that EIB may also be caused by hematogenous or lymphatic spread of viable M. tuberculosis.

\section{Keywords}

Erythema induratum Bazin · Nodular vasculitis - Tuberculid - Cutaneous tuberculosis - Tuberculosis 


\section{Themenverwandte Kasuistiken}

Zuletzt sind in dieser Rubrik folgende themenverwandte Fallberichte veröffentlicht worden:

Elmer A, Bermes U, Drath L, Büscher E, Viertel A (2004) Sepsis und Multiorganversagen nach BCG-Instillation bei Blasenkarzinom. Internist 45: 935-939

Schnaitmann R, Hack M, Gläser F, Schütze B, Tsegai E, Kleber G (2004) Pulmonale Infiltrate bei einem 19-jährigen Patienten mit Dysurie und Hyperkalziämie. Internist 45: 940-945

Siegmund B, Folwaczny C, Bader L et al. (2000) Multiple Muskelabszesse bei tibetanischer Patientin. Internist 41: 903-907

Uerscheln J, Heyers HJ (2001) Intrakraniale epitheloidzellige Granulome mit Progression unter tuberkulostatischer Chemotherapie. Internist 42: 1251-1255

\section{Korrespondierender Autor \\ PD Dr. S. Bassetti}

Klinik für Infektiologie und Spitalhygiene, Universitätsspital

4031 Basel

sbassetti@uhbs.ch

Interessenkonflikt. Es besteht kein Interessenkonflikt. Der korrespondierende Autor versichert, dass keine Verbindungen mit einer Firma, deren Produkt in dem Artikel genannt ist, oder einer Firma, die ein Konkurrenzprodukt vertreibt, bestehen. Die Präsentation des Themas ist unabhängig und die Darstellung der Inhalte produktneutral.

\section{Literatur}

1. Barbagallo J, Tager P, Ingleton R, Hirsch RJ, Weinberg JM (2002) Cutaneous tuberculosis. Diagnosis and treatment. Am J Clin Dermatol 3:319-328

2. Baselga E, Margall N, Barnadas MA, Coll P, de Moragas JM (1997) Detection of Mycobacterium tuberculosis DNA in lobular granulomatous panniculitis (erythema induratum - nodular vasculitis). Arch Dermatol 133: 457-462

3. Chong LY, Kong KLO (1995) Cutaneous tuberculosis in Hong Kong: a 10 year retrospective study. Int J Dermatol 34: 26-29

4. Gawkrodger DJ (1998) Mycobacterial infections. In: Champion RH, Burton JL, Elbling FJG (eds) Text book of dermatology, 6 th edn. Blackwell, London; Vol 2: 1181-1214

5. Kumar B, Muralidhar S (1999) Cutaneous tuberculosis: a twenty-year prospective study. Int J Tuberc Lung Dis 3: $494-500$

6. Manabu H, Kazunori U (2004) Epidemiology of cutaneous tuberculosis in Japan: a retrospective study from 1906-2002. Int J Dermatol 43: 727-731

7. Requena L, Sanchez Yus E (2001) Panniculitis. Part II. Mostly lobular panniculitis. J Am Acad Dermatol 45: 325-361

8. Vieites B, Suárez-Peñaranda JM, Pérez del Molino ML et al. (2005) Recovery of Mycobacterium tuberculosis DNA in biopsies of erythema induratum - results in a series of patients using an improved polymerase chain reaction technique. Br J Dermatol 152: 1360-1398

\section{CME.springer.de}

\section{Neue Serviceangebote}

Im CME.Center auf CME.springer.de stehen Klinikern und Niedergelassenen online über $\mathbf{2 4 0}$ aktuelle und qualitätsgesicherte Fortbildungseinheiten zur Verfügung, die mit jeweils 3 CME-Punkten zertifiziert sind. Um dieses Fortbildungsangebot noch komfortabler zu machen, haben wir unsere Serviceleistungen erweitert:

- Im personalisierten Bereich „Mein CME.Center" haben wir für Sie alle Fortbildungseinheiten zusammengestellt, an denen Sie sofort und ohne weitere Kosten teilnehmen können. Dies sind beispielsweise Fortbildungseinheiten Ihrer abonnierten Zeitschrift und Einheiten, für die Sie bereits ein CME.Ticket eingelöst haben.

- Mit der komfortablen Suchfunktion gelangen Sie noch einfacher zu den gewünschten Fortbildungseinheiten.

- Möchten Sie über den Beitragstitel hinaus weitere Informationen zum Inhalt einer Fortbildungseinheit? Ein Klick genügt und Sie können die Zusammenfassung lesen.

\section{Punkten Sie online!}

Viel Erfolg wünscht Ihnen CME.springer.de

Bei Fragen hilft Ihnen unser Helpdesk gerne weiter CME@springer.com

\section{Hier steht eine Anzeige.}

